\title{
Measurement on properties of MPPC for TOF-PET system
}

\author{
Makoto Yamazaki, ${ }^{*}$ Tohru Takeshita, ${ }^{b}$ and Yoji Hasegawa ${ }^{b}$ \\ a.Interdisciplinary Graduate School of Science and Engineering, Shinshu University, \\ 3-1-1 Asahi, Matsumoto, Japan \\ b.Faculty of Science, Department of Physics, Shinshu University \\ E-mail: yamazaki@azusa.shinshu-u.ac.jp
}

\begin{abstract}
A large number of techniques are rapidly being developed for the diagnosis of cancer, including time-of-flight positron-emission tomography (TOF-PET). To construct an effective TOF-PET system, we introduced lutetium fine silicate (LFS) as a scintillator and a multi-pixel photon counter (MPPC) as a photosensor that exhibits a rapid response. In this study, we investigated four types of MPPCs with a sensitive area of $1 \times 1 \mathrm{~mm}^{2}$ sensitive area and different pitch sizes. All MPPCs showed a timing resolution of $72 \mathrm{ps}$ at the full-width at half-maximum with operating voltages of $\mathrm{dV} \sim 3 \mathrm{~V}$ (with the exception of the 100-pixels MPPC).
\end{abstract}

KEYWORDS: Gamma camera, SPECT, PET PET/CT, coronary CT angiography (CTA); Photon detectors for UV, visible and IR photons (solid-state); Scintillators and scintillating fibres and light guides.

*Corresponding author. 


\section{Contents}

\section{Introduction}

Positron-emission-tomography (PET) systems is highly developing system in medical imaging field. This detects back-to-back gamma rays from the annihilation of a positron and an electron. Especialy for a diagnosis of cancer, the positron is generated by the decay of a nucleus such as fluorine-18 from fluoro-deoxy glucose, a radiopharmaceutical that is injected into body, and accumulates at the cancer site. A pair of photons from an annihilation event is simultaneously captured by detectors surrounding the body. We assume that the location of a cancer is on a line connecting the positions of the detected photons, known as the line of response (LOR). Time-offlight PET (TOF-PET) detects the time difference between the response of a pair of detectors. When the timing resolution of the detectors is optimal, LOR is decreased, and the PET images are enhanced. Therefore, optimal timing resolution is important. To construct a high-resolution PET system, it is necessary for a system to incorporate a scintillator that can rapidly generate scintillation to achieve a short rising time, as well as a photosensor, which achieves a rapid rising response.

For this purpose, we choose lutetium fine silicate (LFS), a newly developed inorganic scintillator [1]. As LFS has a short decay time and high light yield, a good timing resolution is expected. The short decay time typically realizes the short jitter of rising time. Coincidence resolving time (CRT) [2] is determined by the fluctuation of the rising part of signals; thus, the selection of a suitable inorganic scintillator is important.

A multi-pixel photon counter (MPPC), manufactured by Hamamatsu KK, is a silicon-based photosensor that has avalanche photodiodes aligned in a small area [3]. As it is specified to the tolerance of a magnetic field, an MPPC can be used in the setup of magnetic resonance imaging (MRI) as PET-MRI. Recently several types of MPPCs have become available. In this study, we selected MPPCs with 100, 400, 1600, and 2500 pixels in a sensitive area measuring $1 \times 1 \mathrm{~mm}^{2}$. The 2500-pixels MPPC was provided by HAMAMATSU K.K. as an evaluation sample and is not currently available in the market.

In this paper, we evaluate timing resolution as determined by CRT measurements and energy resolution for a detector system consisting of LFS crystals and MPPCs. Based on the results, we discuss the optimal MPPC for a TOF-PET system.

\section{Components and methods}

\subsection{Lutetium Fine Silicate(LFS)}

LFS is an inorganic scintillator manufactured by Zecotek Co. [1] with characteristics similar to those of lutetium yttrium orthosilicate (LYSO). The crystal is highly transparent and the surface is polished. LFS has a short scintillation decay time ( $\sim 36 \mathrm{~ns})$ and a high light output which is three times higher than that of bismuth germanate (BGO). The light output serves as a relative signal amplitude by MPPC according to our measurements (TABLE I). Therefore, it is an ideal material for a TOF-PET system. The properties of LFS are also shown in TABLE I in comparison with BGO and LYSO.

We used an LFS crystal of $3 \times 3 \times 15 \mathrm{~mm}^{2}$ to detect $511-\mathrm{keV}$ annihilation gamma rays. This length will be enough to stop gammas of the energies. 
TABLE I. Scintillators properties

\begin{tabular}{ccccccc}
\hline scintillator & $\begin{array}{c}\text { density } \\
{[\mathbf{g} / \mathbf{c m}}\end{array}$ & $\begin{array}{c}\text { wave } \\
\text { lenghth of } \\
\text { Max. } \\
\text { emission } \\
{[\mathbf{n m}]}\end{array}$ & $\begin{array}{c}\text { attenuatio } \\
\mathbf{n} \\
\text { length } \\
{[\mathbf{c m}]}\end{array}$ & $\begin{array}{c}\text { decay } \\
\text { const. } \\
{[\mathbf{n s}]}\end{array}$ & $\begin{array}{c}\text { refractive } \\
\text { index }\end{array}$ & $\begin{array}{c}\text { relative signal } \\
\text { amplitude with } \\
\text { MPPC }\end{array}$ \\
\hline BGO & 7.1 & 480 & 1.11 & 300 & 2.15 & 1 \\
LYSO & 7.4 & 428 & 1.12 & 44 & 1.82 & 3 \\
LFS & 7.4 & 416 & 1.12 & 35 & 1.81 & 3 \\
\hline
\end{tabular}

\subsection{Multi-Pixel Photon Counter (MPPC)}

The MPPC is a pixelated avalanche photodiode operated in the Geiger mode with a gain greater than $10^{5}$. As each pixel outputs a signal for a photon called a photoelectron, MPPC is regarded as a photon counter. When large numbers of photons reach the sensitive area, the output is the sum of the signals from all fired pixels. MPPC is a semiconducting photosensor; thus, it emits dark noise due to thermal electrons that are predominantly one photoelectron (p.e.) equivalent.

When the PET system maintains a high energy threshold of approximately $400 \mathrm{keV}$, corresponding to tens of photoelectrons for a $1 \times 1 \mathrm{~mm}^{2}$ sensitive area, the dark noise does not degrade performance at all. Therefore it is possible to operate an MPPC at room temperature. Measurements of timing and energy resolution were performed with 100 (S10362-11-100), 400 (S10362-11-050), and 1600 (S10362-11-025) and 2500 (S10943-9428(X))-pixel MPPC, corresponding to pixel pitches of 100,50, 25, and $20 \mu \mathrm{m}$ aligned in an area $1 \times 1 \mathrm{~mm}^{2}$. The pixel size is expected to affect the CRT because the capacitance of a pixel reduces with pixel pitch.

The timing and energy resolution for these four types of MPPCs coupled with an LFS were measured as a function of pixel pitch and gain.

The characteristics of the MPPCs used in this study are summarized in TABLE II.

\begin{tabular}{ccccc}
\hline $\begin{array}{l}\text { number } \\
\text { pixels }\end{array}$ & pixel pitch $[\boldsymbol{\mu m}]$ & gain $(\times \mathbf{1 0}$ & capacitance $[\mathbf{f F}]$ & $\begin{array}{c}\text { Dark noise } \\
{[\mathbf{k H z}]} \\
(>\mathbf{1 ~ p . e} .)\end{array}$ \\
\hline $\mathbf{1 0 0}$ & 100 & 24.1 & 380 & 600 \\
$\mathbf{4 0 0}$ & 50 & 7.50 & 97 & 400 \\
$\mathbf{1 6 0 0}$ & 25 & 2.74 & 17 & 300 \\
$\mathbf{2 5 0 0}$ & 20 & 2.03 & 12 & 40 \\
\hline
\end{tabular}

TABLEII. Characteristics of some MPPCs which have different pixel pitches. The dark noise is typical at room temperature. 


\subsection{Methods}

Initially, gain was measured as a function of the bias voltage. A light-emitting diode was employed, and lights were directly detected by each MPPC to count the number of photoelectrons. To estimate the gain an MPPC was operated at several bias voltages above the

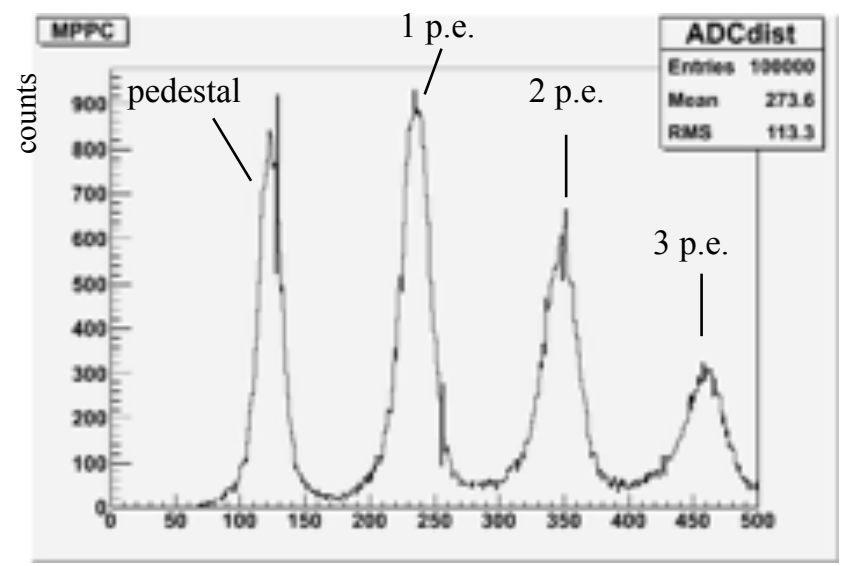

Fig.1 Each photoelectron's peak can be separated in ADC distribution. The data were taken at the gain of $3.0 \times 10^{5}$ with 1600-pixels MPPC.

break-down voltage. The distribution of signal amplitude measured using an analog-to-digital convertor (ADC) shows the photoelectrons peaks (Fig. 1). The position of one and two photoelectrons peaks are determined by fitting the distribution to Gaussian functions, and the difference between them is calculated in a unit of an ADC channel. Gain is calculated according to Equation (1).

$$
\text { Gain }=\frac{\text { ADCchannels } \times 0.25 \mathrm{pC} / \mathrm{ch}}{1.602 \times 10^{-19} \mathrm{C} \times(\text { Amp.gain })} \ldots \ldots(1)
$$

The schematic view of the CRT measurement and energy resolution setup is shown in Fig. 2. Each LFS is wrapped in a reflection film (98\% reflectance, Kimoto [4]) and is directly coupled with an MPPC without any glue. Two detectors are aligned in a face-to-face configuration to detect annihilation gamma rays from the ${ }^{22} \mathrm{Na}$ source. 


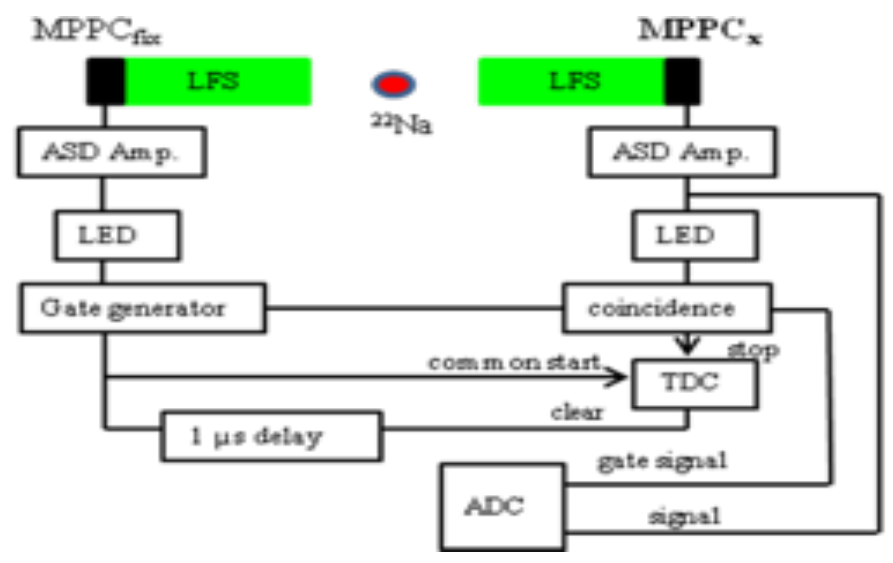

Fig. 2 Experimental setup

Signals from each MPPC were fed into an amplifier shaper discriminator (ASD), originally developed for thin gap chambers in an LHC/ATLAS experiment [5]. According to our measurements the ASD has a gain of 595. The analog signals from the ASD were led to a leading-edge discriminator shown in Fig. 2 as LEDs. In this study, a constant-fraction discriminator was not employed so that we could analyze jitter of signals from detectors with an LFS and an MPPC to determin the timing resolution from the scientific point of view. An MPPC of 1600 pixels, which is defined as MPPC fix in Fig. 2, was used as one of the two detectors and generates the start signals for the time-to-digital convertor (TDC). The MPPC fix signals from the leading-edge discriminator were transmitted to a gate generator, and a coincidence module. The other detector employed 100, 400, 1600, 2500-pixels MPPCs indicated by $\mathrm{MPPC}_{\mathrm{x}}$ in Fig. 2 and the signals from the leading-edge discriminator was fed into a coincidence module. The width of the MPPC fix signal from the gate generator was expanded and the signals from $M P P C_{x}$ were delayed so that each signal pulse from $\mathrm{MPPC}_{\mathrm{x}}$ was always emitted within the time window of the corresponding pulse from the $\mathrm{MPPC}_{\text {fix }}$. A TDC acquires a start signal from $\mathrm{MPPC}_{\text {fix }}$ and a stop signal from the coincidence module. This coincidence module outputs a signal in the case of two input signals overlapping in time; therefore, it outputs a signal when the MPPC $\mathrm{x}_{\mathrm{x}}$ signal arrives.

To measure the energy resolution of each MPPC for $511-\mathrm{keV}$ annihilation gamma rays the signal from the detector was led to the charge sensitive analog-to-digital convertor (ADC). This $\mathrm{ADC}$ offers a resolution of 12 bits whose input sensitivity of the ADC is $0.25 \mathrm{pC}$ / channel for a full-scale range of $1024 \mathrm{pC}$. The coincidence output was also used as the gate signal of the ADC to corroborate the measurement of $511-\mathrm{keV}$ back to back gamma rays. The time width of the gate signal was set to $300 \mathrm{~ns}$ so that it entirely covered the raw signal pulse from $\mathrm{MPPC}_{\mathrm{x}}$.

\section{Results}

Gain was measured as a function applied bias voltage; then, the breakdown voltage was determined at the vertex of the gain line and the horizontal line at a gain equal to 0 , as shown in 


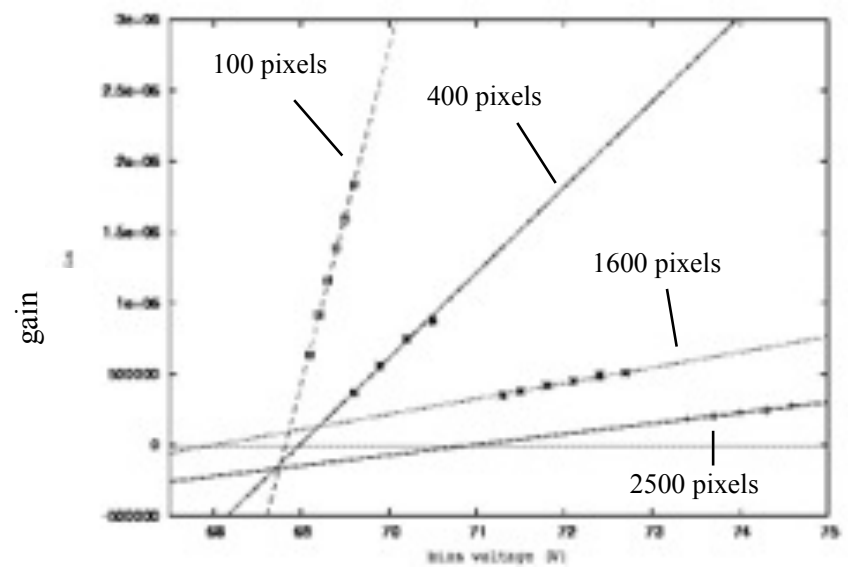

Fig. 3 Gains for four types of MPPC are showr bias voltage [V] iperating bias voltage. Data points for each MPPC are fitted to a linear function deriving the breakdown voltage.
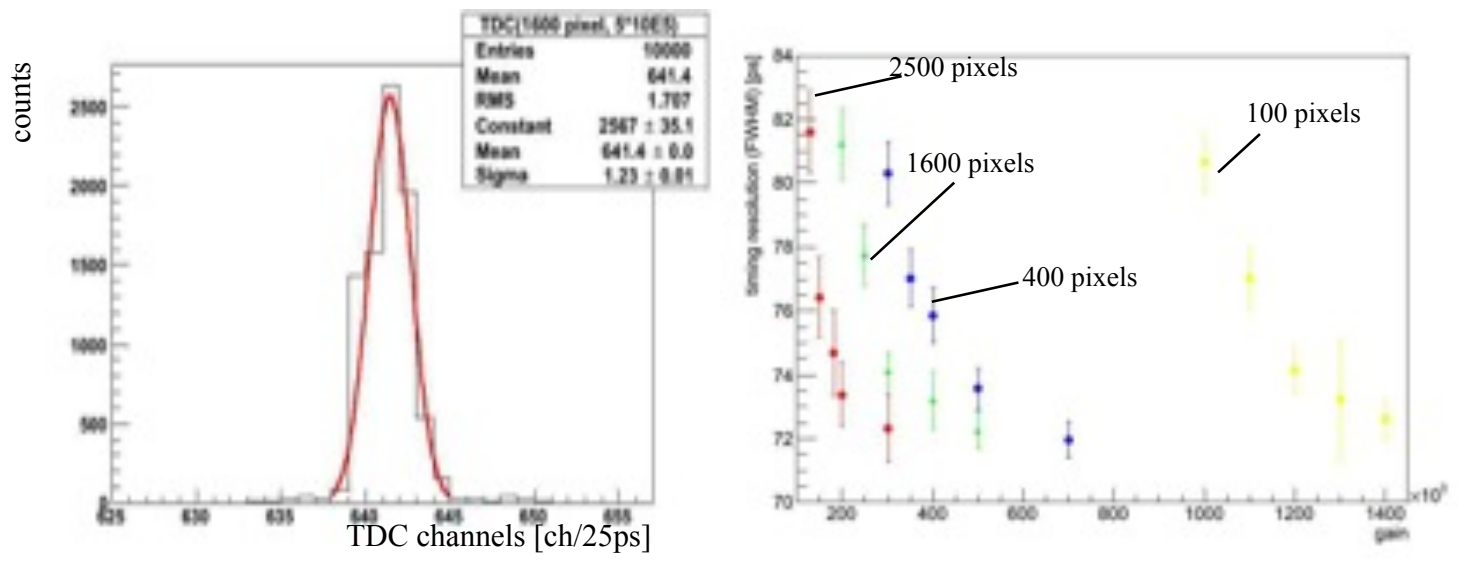

Fig. 4 (a) TDC distribution of the MPPC with 1600 pixels in a $1 \times 1 \mathrm{~mm}^{2}$ sensitive area is fitted by Gaussian function. The timing resolution is evaluated as the FWHM unit from the standard deviation (sigma). (b) Timing resolution plotted as a function of gains for four types of MPPCs.

Fig. 3. The breakdown voltages of $68.8,69.0,68.0$ and $71.0 \mathrm{~V}$ for 100, 400, and 1600-pixel MPPCs, respectively, were obtained.

To measure a CRT, TDC distributions of $\mathrm{MPPC}_{\mathrm{x}}(\mathrm{x}=100,400,1600$, and 2500 pixels $)$ were fitted using a Gaussian function. For example, the result of MPPC 1600 is shown in Fig. 4 (a) when the operating bias is set at a value to achieve a gain of $5.0 \times 10^{5}$. The internal time resolution of the TDC module is 25 ps per channel, therefore the timing resolution for $\mathrm{MPPC}_{1600}$ was $72.3 \pm 0.6 \mathrm{ps}$ at FWHM.

For the other MPPCs, the same procedure was under taken to estimate the timing resolution at several gain values. Fig. 4 (b) shows timing resolution as a function of gain. This result indicates that the timing resolution improves following a gain increase.

Energy resolution resulted in an ADC distribution. The ADC distribution for the 1600-pixel MPPC in which the operation voltage was set to achieve a gain of $3.0 \times 10^{5}$, and the 

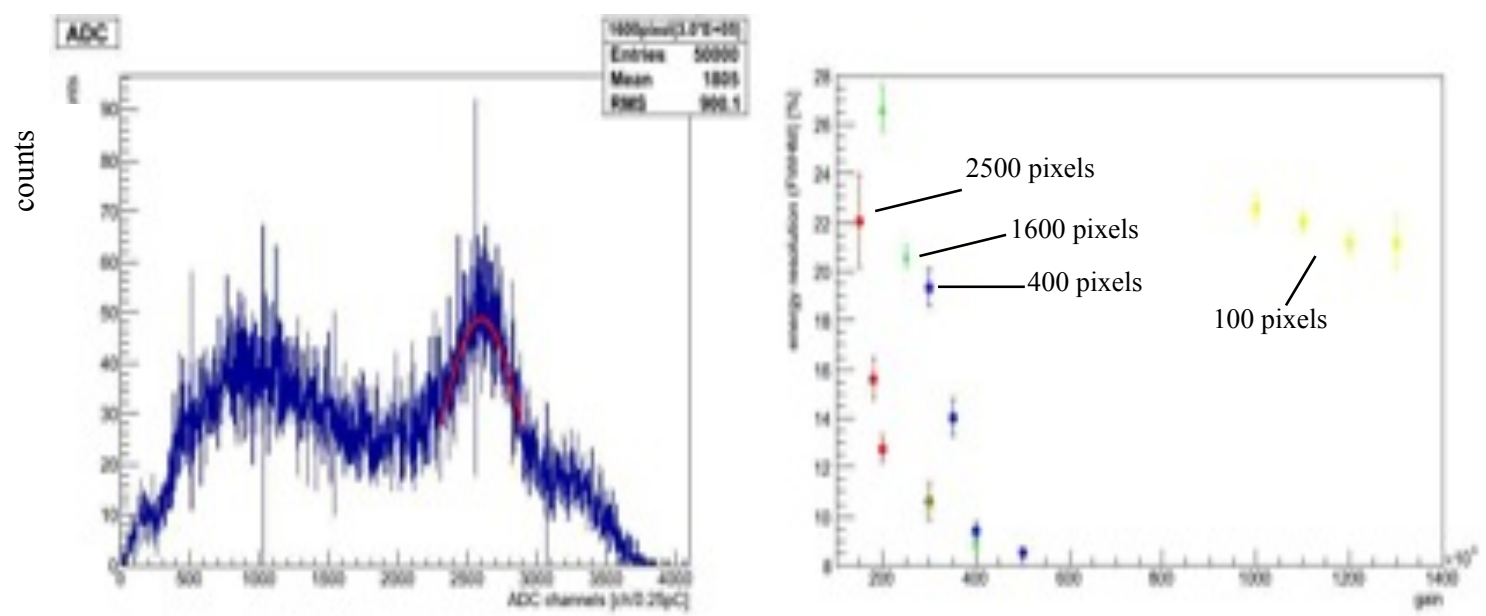

$\mathrm{ADC}$ channels $[\mathrm{ch} / 0.25 \mathrm{pC}]$

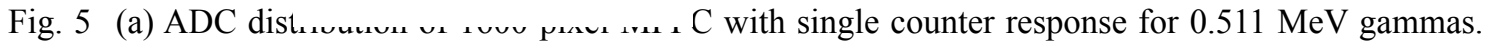
The $0.511 \mathrm{MeV}$ peak was fitted by Gaussian curve (red line), while the compton events are seen at lower energies. (b) Energy resolution for MPPCs as a function of gain.

annihilation gamma ray peak was fitted to a Gaussian function resulted in an energy resolution of $10.6 \pm 0.2 \%$ at FWHM [Fig. 5 (a)]. Energy resolution as a function of gain is plotted in Fig. 5 (b) for every MPPC.

TABLE III summarizes the timing and energy resolution of four types of MPPC. The values listed in TABLE III were measured at a gain of $3.0 \times 10^{5}$ except for the 100-pixels MPPC. The 100-pixels MPPC has a high gain by its nature; therefore, the energy and timing resolutions cannot be compared with those of other MPPCs at the same gain. The values of the 100-pixel MPPC at a gain of $10.0 \times 10^{5}$ are shown in TABLEIII.

TABLE III. Energy and timing resolution

\begin{tabular}{cccc}
\hline $\begin{array}{c}\text { number of } \\
\text { pixels }\end{array}$ & gain & $\begin{array}{c}\text { energy resolution } \\
{[\%]}\end{array}$ & $\begin{array}{c}\text { timing resolution (FWHM) } \\
{[\mathbf{p s}]}\end{array}$ \\
\hline $\mathbf{1 0 0}$ & $10.0 \times 10$ & $21.1 \pm 0.9$ & $80.7 \pm 0.9$ \\
$\mathbf{4 0 0}$ & $3.0 \times 10$ & $19.4 \pm 0.7$ & $80.3 \pm 1.0$ \\
$\mathbf{1 6 0 0}$ & $3.0 \times 10$ & $10.5 \pm 0.2$ & $74.1 \pm 0.6$ \\
$\mathbf{2 5 0 0}$ & $3.0 \times 10$ & $10.6 \pm 0.7$ & $72.3 \pm 1.1$ \\
\hline
\end{tabular}

\section{Discussion}

In this study, we measured the energy and timing resolutions for four types of MPPCs with different numbers of pixels. In addition, the resolution of each MPPC was measured by changing the gain to search for a suitable photosensor for a next-generation PET system. Improved resolutions are achieved at high gains.

We commonly operate an MPPC at a bias of $2 \mathrm{~V}$ higher than the breakdown voltage. In this case, an MPPC can be easily controlled. However, the gain of MPPC 100 significantly depends on its bias voltage; therefore, it is difficult to compare with other MPPCs, which makes stable operation difficult. The bias voltage should be controlled within less than $0.05 \mathrm{~V}$. Thus MPPC 100 will not be suitable for next-generation PET system.

The timing resolution is very important in next-generation PET systems to obtain a clear and high-contrast image. Therefore, we measured CRT with a leading-edge discriminator. A leading- 
edge discriminator outputs a digital signal when the input signal exceeds the threshold level. In particular, the rising part of a signal determines the timing of the output. Optimal timing resolution for each MPPC was achieved at a higher gain, although such a high gain is not required for normal usage. An leading-edge discriminator was used to analyze the effect of the rising part of the signals, which takes the time jitter of the leading part of a signal as the object of timing resolution. This means that the trailing part of a signal is not relevant in this measurement. The fast rising time of the signal could contribute to a good timing resolution, while the short jitter of the leading signal leads to a short CRT. Fig. 6 schematically shows the reason for the improvement in timing resolution by the fast rising time. In particular, the scintillator that has fast decay time generates a fast rising-time signal. In terms of theory [6], the light intensity of an inorganic scintillator follows the relation defined in Equation (2),

$$
I(t)=I_{0}\left(e^{-t / \tau_{d}}-e^{-t / \tau_{r}}\right) \cdots \cdots \cdots(2)
$$

where $I_{0}$ is initial intensity of a signal, and $\tau_{d}$ and $\tau_{\mathrm{r}}$ are the decay time and rise time of scintillation, respectively. The maximum intensity depends on the ratio of $\tau_{\mathrm{d}}$ and $\tau_{\mathrm{r}}$.

An inorganic scintillator LFS, which has recently become available in the market, has a remarkably fast decay time of approximately 35 ns. In addition, MPPCs also respond very rapidly, and their rising time is a few nano seconds. These features suggest a good timing resolution. In addition, the time jitter of the leading part depends on the pulse height, which is also shown in Fig. 6. A high yield of scintillation light increases the signal amplitude, resulting in a faster rising time. In Fig. 6, two pulses with different heights are compared along the vertical line. A leading-edge discriminator detects the leading part of the signal; thus, the time jitter which is a fluctuation of rising time of a signal reduces when the signal increases. Therefore, in general, timing resolution improves at a higher gain.

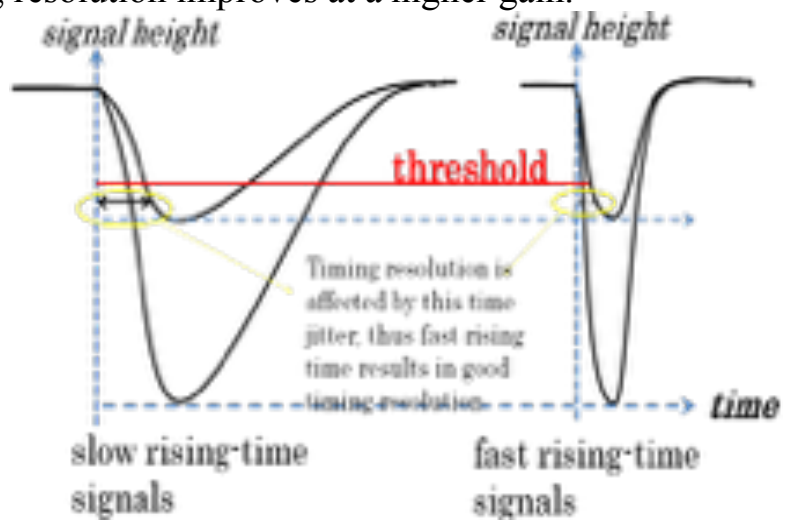

Fig. 6 Differences of time jitter of low and fast rising-time signals.

According to TABLE III, the energy resolutions of scintillators with 20- and $25-\mu \mathrm{m}$-pitch MPPCs are almost equal. In terms of other aspects of energy resolution, this could achieve a good resolution at higher gain, as shown in Fig. 5 (b). The photon detection efficiency (PDE) increases according to the bias voltage, thus improving energy resolution.

\section{Limitation}

Since we are interested in the measurement of CRT, the leading part of the signal is concerned. The fluctuation of them affects to the timing resolution, although the number of lights detected by $0.511 \mathrm{MeV}$ gamma rays is almost constant about 70 photoelectrons in this configuration. On the other hand, the measurements with constant fraction discriminator (CFD) will give the same timing signals independent to the pulse height of the signals. Therefore we employed the leading edge discriminator for the measurement of CRT. 
Many groups are investigating PET systems using PC-based systems, while we employ NIM / CAMAC modules. The main aim of this study was to apply the technology of high-energy physics to the PET system, thus our modules are constructed for measuring up to high-energy experiments. However, we demonstrated that the systems have significant potential when for use as a next-generation PET system especially for TOF-PET. We also performed measurements of the electronic-timing resolution using a clock generator to understand the limitations of the system. The same timing signals from the clock generator were input into the circuit instead of LFS-MPPC detectors in Fig. 2 as an alternative. One signal was delayed for the arrival time of the other signal. The timing resolution of our electronics is depicted in Fig. 7. This indicates that our electronics have a small intrinsic timing fluctuation of $34.5 \pm 0.3 \mathrm{ps}$ at FWHM which is much smaller than our results.

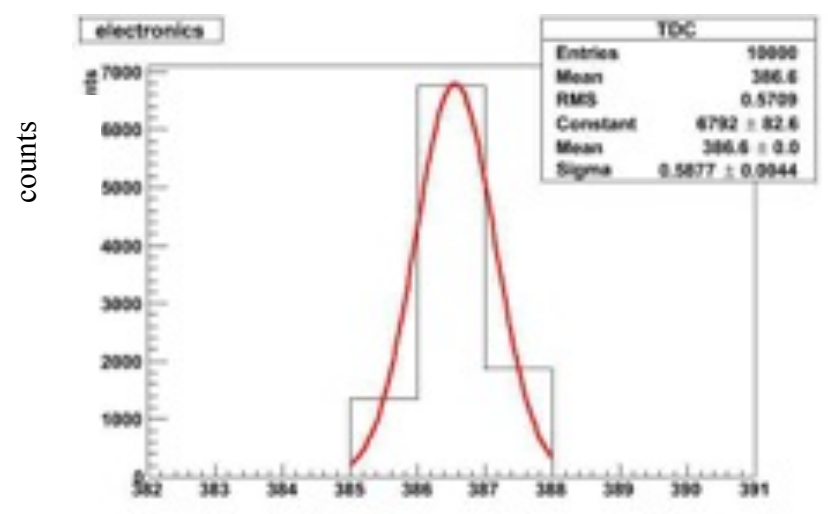

\section{Future prospect}

Fig. 7 Timing resolution of electronics measured using signals from the clock

We used an LFS inorganic crystal with a surface area of $3 \times 3$ m menatertaghed taran showe that

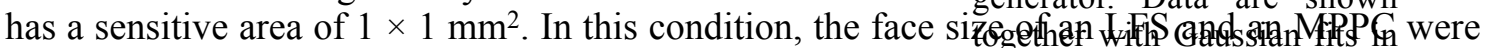
not compatible, and leakage of photons must be addressథdodistnibithor MPPCs when constructing array of detectors which offer couples of an LFS and an MPPC. Therefore, in the future, we will employ an LFS with a surface area of $3 \times 3 \mathrm{~mm}^{2}$ to fit with the outer size of the package of MPPC. Such a device with a size of $2.4 \times 1.9 \mathrm{~mm}^{2}$ is known as a surface mount detector. When constructing an array of MPPCs, the surface size of the LFS and the MPPC must match one to one, because we asSume that achdeted chor consists of an optically separated LFS and an MPPC to determine the position of a single $511-\mathrm{keV}$ gamma ray without the use of an Anger calculation. If the lights from the matrix of scintillators are readout in case that the number of photosensors is smaller than that of scintillators such that light from several scintillators is captured by single photosensor, an Anger calculation must be required. It may degrade the spatial resolution. Our technique to attach a scintillator to a photosensor does not need Anger calculation; therefore the system has a simple readout and the determination of incident gamma ray position is straightforward. Furthermore, as the number of photoelectrons detected by the 1 $\times 1 \mathrm{~mm}^{2}$ MPPC is sufficient to detect the $0.511 \mathrm{MeV}$ peak, our approach with a smaller photosensor is verified.

\section{Conclusion}

We measured timing and energy resolution with an inorganic scintillator an LFS and MPPCs, which have several types of pixel pitches. All MPPCs achieved a timing resolution of $72 \mathrm{ps}$ at FWHM at higher gains. The jitter of electronics was $35 \mathrm{ps}$; therefore, the LFS-MPPC coupling detector is able to detect $511-\mathrm{keV}$ back-to-back gamma rays. The energy resolution of $10 \%$ of $\sigma$ / E for 511-keV gamma rays was obtained for the MPPC with a $25-\mu \mathrm{m}$ and $50-\mu \mathrm{m}$ pixel pitch. Based on these results, we conclude that using the MPPC, which has a larger number of pixels 
than 1600, is suitable for next-generation TOF-PET systems. In particular, the timing resolution reduces image noise, which is currently an issue of the TOF-PET system. This study supports the development of a PET system in this setting.

\section{Acknowledgement}

This work was supported by JSPS KAKENHI Grant-in-Aid for Scientific Research (C) Number 23602007.

\section{Reference}

[1] Zecotek Photonics Inc., http://www.zecotek.com/

[2] S. Seifert, R. Vinke, H. T. van Dam, H. Lohner, P. Dendooven, F. J. Beekman, D. R. Schaart, Ultra precise timing with SiPM-based TOF PET scintillation detectors, Proceedings of the Nuclear Science Symposium Conference Record (NSS/MIC), p. 2329, Orlando, FL,Oct. 24 2009-Nov. 1, 2009

[3] Hamamatsu Photonics K.K., http://jp.hamamatsu.com/

[4] Kimoto Co. Ltd., http://www.kimoto.co.jp/

[5] O. Sasaki and M. Yoshida, ASD IC for the Thin Gap Chambers in the LHC Atlas Experiment, IEEE Trans. Nucl. Sci., 46 (1999), p.1871

[6] S. Seifert, J.H.L. Steenbergen, H.T. van Dam and D.R. Schaart, Accurate measurement of the rise and decay times of fast scintillators with solid state photon counters, JINST 7, p09004, 2012

[7] L. Papadopoulos, Rising time of scintillation emission in inorganic and organic scintillators, Nuclear Instruments and Methods in physics research A 401 (1997) 322-328

[8] S.E. Derenzo, M.J. Weber, W.W. Moses and C. Dujardin, Measurements of the Intrinsic Rise Time of Common Inorganic Scintillators, IEEE Trans Nucl Sci, NS-47, p.860-864, 2000

[9] Makoto Yamazaki, Tohru Takeshita and Yoji Hasegawa, Next-Generation PET Capability with Lutetium Fine Silicate and Multi-Pixel Photon Counter, JINST 7, p.10014, 2012 